\title{
Original
}

\section{Evaluating the Bone Regeneration of nHAp/PPC Membrane in Rabbit Calvarial Defect}

\author{
Chuanjian Lan ${ }^{1,2)}$, Xingchen Xiang ${ }^{3)}, \mathrm{Xi} \mathrm{He}^{1)}$, Yongsheng Pan ${ }^{1)}$, Duo Sun ${ }^{1)}$ and Jiang $\mathrm{Li}^{1)}$ \\ 1) Department of Prosthodontics, School and Hospital of Stomatology, Jilin University, Changchun, China \\ 2) Jilin Provincial Key Laboratory of Tooth Development and Bone Remodeling (School and Hospital of Stomatology, Jilin University), Changchun, \\ China \\ 3) Department of General Dentistry, School and Hospital of Stomatology, Jilin University, Changchun, China \\ (Accepted for publication, October 3, 2019)
}

\begin{abstract}
This study aims to evaluate the ability of poly(propylene carbonate) (PPC)-loaded nanohydroxyapatite (nHAp) membrane to regenerate bones. Methods: Six New Zealand white rabbits were randomly and equally divided into two groups. Four $6 \mathrm{~mm}$ diameter transosseous round defects were made at the parietal bone of each rabbit. One defect was not covered with the membrane, and the others were covered with $10 \%$ or $20 \% \mathrm{nHAp} / \mathrm{PPC}$ membrane or HEAL-ALL membrane. Animals of each group were sacrificed at the 4 and 12 weeks after the operation, respectively. The rabbit parietal bones were removed for radiological and histological evaluation. Results: Micro-computed tomography results showed that the bone regeneration of the experimental group was significantly higher than that of the control group in the absence of bone powder after 4 and 12 weeks, and the $20 \% \mathrm{nHAp} / \mathrm{PPC}$ group showed the most significant effect on bone regeneration. Histological analysis found that the nHAp/PPC membranes and HEAL-ALL membranes of the experimental groups prevent the growth of soft tissues during bone regeneration. In summary, the nHAp/PPC membrane showed good biocompatibility during the repair of rabbit parietal defects, and the $20 \% \mathrm{nHAp} / \mathrm{PPC}$ membrane is prominent.
\end{abstract}

Key words: Poly(propylene carbonate), Nanohydroxyapatite, Guided bone regeneration technique, Micro-computed tomography

\section{Introduction}

Guided bone regeneration (GBR) has been widely used in clinical practice $^{1,2)}$ and is the most commonly used technique for restoring alveolar bone defects and treating bone defects around implants ${ }^{3,4)}$. GBR uses the membrane as a barrier to prevent connective tissues and mucosal epithelial cells from growing into the defects ${ }^{5,6}$, thereby allowing underlying bones to regenerate. Currently, various GBR membranes have been developed, which can be divided into non-absorbable and absorbable membrane ${ }^{7}$. The expanded polytetrafluoroethylene membrane is the first non-absorbable GBR membrane. Titanium alloys have been widely used as non-absorbable GBR membranes. Non-absorbable GBR membranes have good biocompatibility and space maintenance ${ }^{8)}$ and are mostly used in vertical bone defects ${ }^{9}$. However, the disadvantages of non-absorbable membrane are prominent. Several studies have shown that non-absorbable GBR membranes are prone to result in soft tissue exposure $^{10)}$ because of the excessive tension generated by soft tissues. Moreover, a second surgery is usually needed to remove a non-absorbable membrane completely. Meanwhile, absorbable GBR membranes, which are considered patient-friendly barrier membranes, are self-degradable, and their removal does not require secondary surgery ${ }^{11)}$. Currently, most clinically used absorbable membranes are collagen components. However, despite their advantages, absorbable GBR membranes have poor strength and rapidly degrade. Therefore, developing a novel absorbable GBR membrane is necessary.

Poly(propylene carbonate) (PPC) is an aliphatic polyester with good

Correspondence to: Dr. Jiang Li, Department of Prosthodontics, School and Hospital of Stomatology, Jilin University, Changchun 130021, China; Tel: +86-0431-88796018; E-mail: 444706646@qq.com biocompatibility and biodegradability ${ }^{12-14)}$ and can be used as a medical material ${ }^{15)}$. Nanohydroxyapatite (nHAp) is the main inorganic component in bone tissues and has good biocompatibility ${ }^{16,17}$. Additionally, nHAp plays an important role in bone cell differentiation and mineralization and promotes bone growth ${ }^{18,19}$. In our previous study, nHAp and PPC were mixed into an nHAp/PPC GBR membrane by solvent casting/ particulate leaching method, and the mechanical properties and cell compatibility of the resulting membrane were studied ${ }^{20,21)}$. The results indicated that $\mathrm{nHAp} / \mathrm{PPC}$ membrane is a biocompatible membrane $\mathrm{e}^{20,21)}$ that promotes the proliferation and differentiation of MG63 cells ${ }^{21)}$. The nHAp/PPC membrane containing $10 \%$ and $20 \%$ nHAp by weight exhibited excellent mechanical properties. To further explore the feasibility of nHAp/PPC membrane as a guide membrane for bone regeneration, this study evaluated the effect of $10 \%$ and $20 \% \mathrm{nHAp} / \mathrm{PPC}$ groups on bone defect repair in vivo. Moreover, HEAL-ALL membrane, which is a resorbable membrane for clinical applications, is used as positive control.

\section{Materials and Methods \\ Preparation of $n H A p / P P C$ GBR and HEAL-ALL membranes}

The nHAp/PPC membranes were prepared according to the solvent casting/particulate leaching method used in our previous experiments ${ }^{20)}$. Briefly, nHAp particles were added to a chloroform solution containing PPC, and the resulting solution was mixed and poured into a glass petri dish containing $\mathrm{NaCl}$ particles. After the chloroform was volatilized, the remaining material was soaked in deionized water for the removal of the $\mathrm{NaCl}$ particles.

The prepared $10 \%$ and $20 \% \mathrm{nHAp} / \mathrm{PPC}$ membranes and the HEALALL membranes (Yan TaiZhenghai Biotechnology Corporation, 


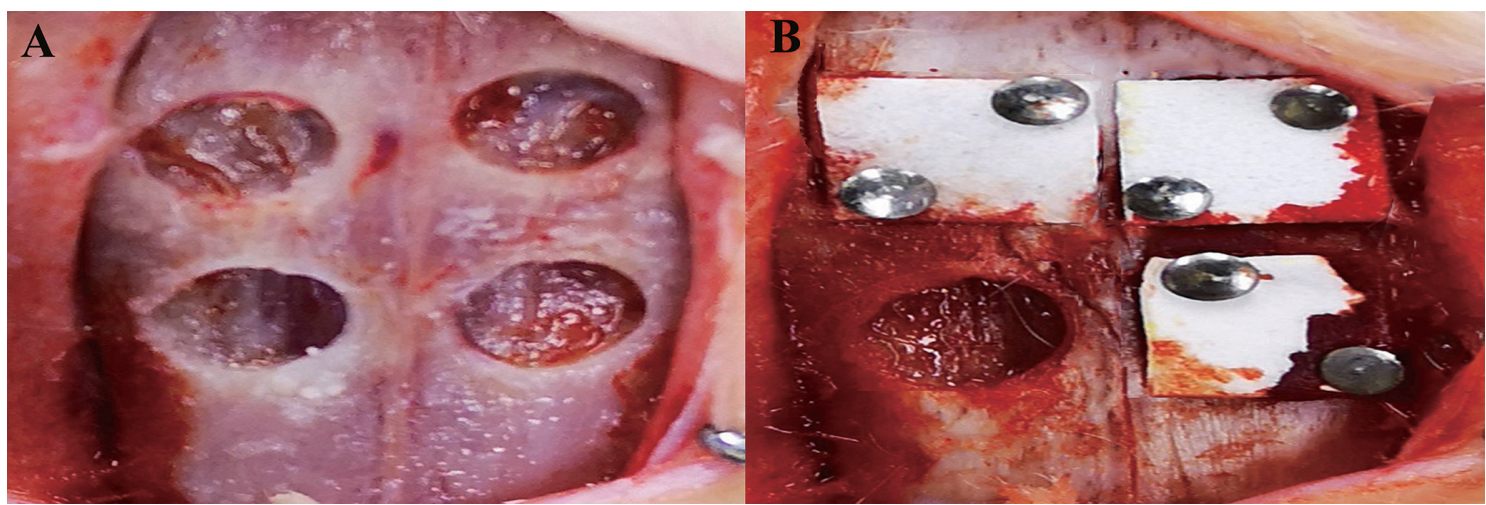

Figure 1. Surgical procedure. A: Four defects $(\varnothing=6 \mathrm{~mm})$ in the calvarium of 6 rabbits. B: Random assignment of defects, control group (left down), $10 \% \mathrm{nHAp} / \mathrm{PPC}$ group (left top), $20 \% \mathrm{nHAp} / \mathrm{PPC}$ (right top), and HEAL-ALL (right down).

Table 1. Micro-CT tomography morphometric parameters of bone

\begin{tabular}{cclc}
\hline Abbreviation & \multicolumn{1}{c}{ Parameter } & \multicolumn{1}{c}{ Explanation } & Unit \\
\hline BV/TV & Bone volume fraction & $\begin{array}{l}\text { Ratio of bone volume to total } \\
\text { volume in a region of interest }\end{array}$ & $\%$ \\
\hline Tb.N & Trabecular number & $\begin{array}{l}\text { Average number of intersections } \\
\text { between bone and non-bone tissues } \\
\text { per mm }\end{array}$ & $1 / \mathrm{mm}$ \\
\hline Tb.Th & Trabecular thickness & $\begin{array}{l}\text { Average thickness of the trabecular } \\
\text { Bone }\end{array}$ & $\mathrm{mm}$ \\
\hline Tb.Sp & Trabecular separation & $\begin{array}{l}\text { Average width of the medullary } \\
\text { between the trabeculae }\end{array}$ & $\mathrm{mm}$ \\
\hline
\end{tabular}

Shandong, China) were cut into squares of $7 \mathrm{~mm} \times 7 \mathrm{~mm}$ size. Then, the membranes were used for surgery after sterilization.

\section{Animal and surgical procedure}

Six adult male New Zealand white rabbits (Yisi Experimental Animal Technology Co., Ltd., Changchun, China, license number: SCXK [Ji]-2016-0004) weighing 2.5-3.5 kg were randomly and equally divided into two groups. The animal experiments were approved by the ethics committee of Jilin University (No. 201825). The rabbits were fed separately in cages by the animal experiment center of basic medical college of Jilin University. The animals were fed uniformly for 1 week before the operation for them to adapt to the new environment and were fasted on water for $12 \mathrm{~h}$ before the operation.

The surgical procedures are shown in Fig. 1. The rabbits were weighed and administered with intramuscular anesthesia SuMianXin II $(0.1 \mathrm{ml} / \mathrm{kg}$ to $0.2 \mathrm{ml} / \mathrm{kg}$, intramuscularly, Quartermaster University of PLA, Changchun, China, Military Veterinary Institute). In each rabbit, local anesthesia was used to infiltrate the operative area ( $2 \%$ lidocaine, $1: 100,000$ epinephrine), and hair covering the skull of the rabbit was cut. The surgical area was disinfected with iodophor disinfectant, and a longitudinal incision of approximately $3 \mathrm{~cm}$ in size was made from the nasal bone to the occipital bone to remove the periosteum and expose the parietal bone. After washing with normal saline, a bone drill was used to make four round $6 \mathrm{~mm}$ bone defects that did not damage the dura. Care was exercised to avoid injury of the dura. Two defects were formed on each side of the middle cranial suture. The spacing between each adjacent bone defect was more than $2 \mathrm{~mm}$ (Fig. 1A). Three bone defects were covered with $10 \% \mathrm{nHAp} / \mathrm{PPC}$ membrane, $20 \% \mathrm{nHAp} / \mathrm{PPC}$ membrane, and HEAL-ALL membrane, respectively. Each membrane was fixed with titanium nails. The remaining defect was left without membrane (Fig. 1B). The flaps were then repositioned without tension and sutured with a 4-0 suture material. Lastly, the surgical sites were sutured, and the animals received antibiotics (penicillin, 100,000 U/days) for 3 days. Each group comprising three animals was sacrificed at the 4 and 12 weeks after surgery.

\section{Clinical observations}

The animals were carefully observed and evaluated for inflammation, allergic reactions, and other complications around the surgical site 4 and 12 weeks after surgery.

\section{Micro-computed tomography (CT) Evaluation}

The parietal bone containing the healed sites were surgically harvested and immediately fixed in neutral buffered formalin (10\%) for 48 h. Each parietal bone was scanned for new bone formation at $50 \mathrm{kV}, 800$ $\mu \mathrm{m}, 1,304 \mu \mathrm{m} \times 1,024 \mu \mathrm{m}$ with Micro-CT scanners (Skyscan 1076, Force, Belgium). The four $6 \mathrm{~mm} \times 6 \mathrm{~mm}$ regions on the middle line of the skull were set as areas of interest for the three-dimensional reconstruction (region of interest). The threshold was selected according to specimen-specific thresholds ${ }^{22}$. The volume of interest (VOI) was selected corresponding to the dimensions of the defect sites, with 6-mm full-thickness cylinders for measurement of mineralized tissue volume and mineralized tissue volume was measured in VOI. N-recon software was used for three-dimensional image reconstruction. Basing on the Micro-CT images, CT-AN software (Amira, Visualization Sciences Group, Düsseldorf, Germany) analyzed the formation of new bones and trabecular bones (Table 1).

\section{Histologic analysis}

After Micro-CT analysis, each parietal bone was decalcified in a glass vial containing $30 \mathrm{ml}$ of $10 \%$ ethylene diamine tetraacetic acid (EDTA) solution for 21 days. The EDTA solution was changed daily. 


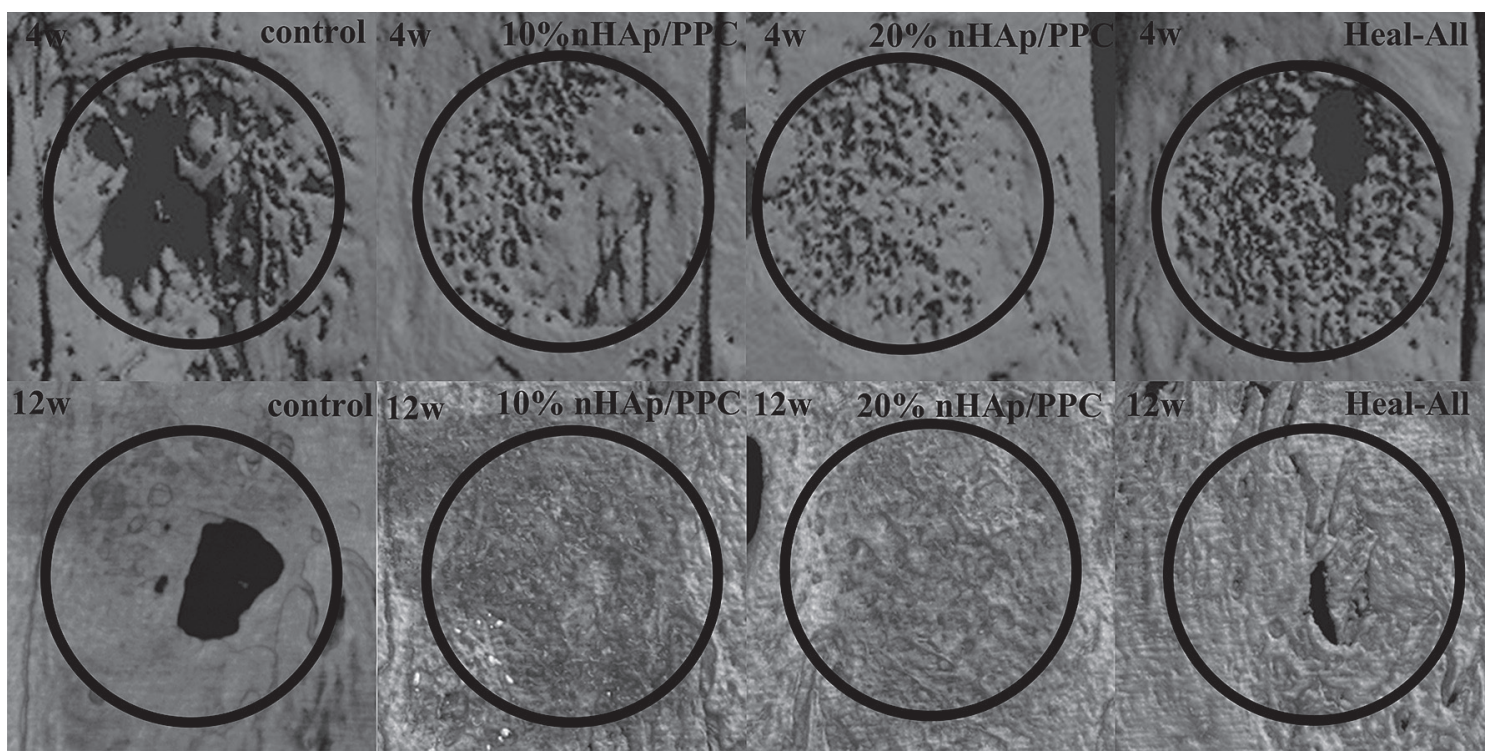

Figure 2. 3D reconstructed micro-CT images of defects at 4 and 12 weeks.

Then, the parietal bone was subjected to ethanol gradient dehydration, wax penetration, and embedding. The embedded wax block was placed in a $-20{ }^{\circ} \mathrm{C}$ refrigerator and frozen for more than $2 \mathrm{~h}$. The embedded block was then placed in a room temperature environment for future use. A $5 \mu \mathrm{m}$-thick tissue section was cut in the middle of the bone defect. Then, the tissue section was stained with hematoxylin-eosin. The stained histologic slides were examined with a light microscope (Olympus BX41, Tokyo, Japan) equipped with a camera (Olympus DP70, Tokyo, Japan).

\section{Statistical analysis}

All data were initially analyzed to test their normality using the Shapiro-Wilk test. Independent sample t-test and one-way analysis of variance (ANOVA) ( $\mathrm{p}$ value $<0.05$ was considered significant) were performed in SPSS 22 software. The data were presented as means \pm standard deviation.

\section{Results}

\section{Clinical observations}

All rabbits survived the operation in good health without any abnormal conditions. No bleeding, inflammation, swelling, and other postoperative complications were observed. After 4 and 12 weeks, the skin of each group healed well, and the tissue and membranes were not exposed.

\section{Micro-CT analysis}

The three-dimensional reconstruction images of the rabbit parietal bone 4 and 12 weeks after surgery are illustrated in Fig. 2. Four weeks after surgery, the defect edges of the four defects in the absence of bone powder were visible although bone regeneration was observed. In the control group, a large number of bone defects were not repaired. In the $10 \%$ and $20 \% \mathrm{nHAp} / \mathrm{PPC}$ and HEAL-ALL groups, the repair ranges of the bone defects were higher than those of the control group. Meanwhile, 12 weeks after surgery, the extent of bone repair in the four groups of defects increased. The $10 \%$ and $20 \% \mathrm{nHAp} / \mathrm{PPC}$ groups showed higher extent of bone repair than the other groups.

The ratio of bone volume and total volume in each of the affected areas 4 and 12 weeks after surgery is displayed in Fig. 3. The BV/TV

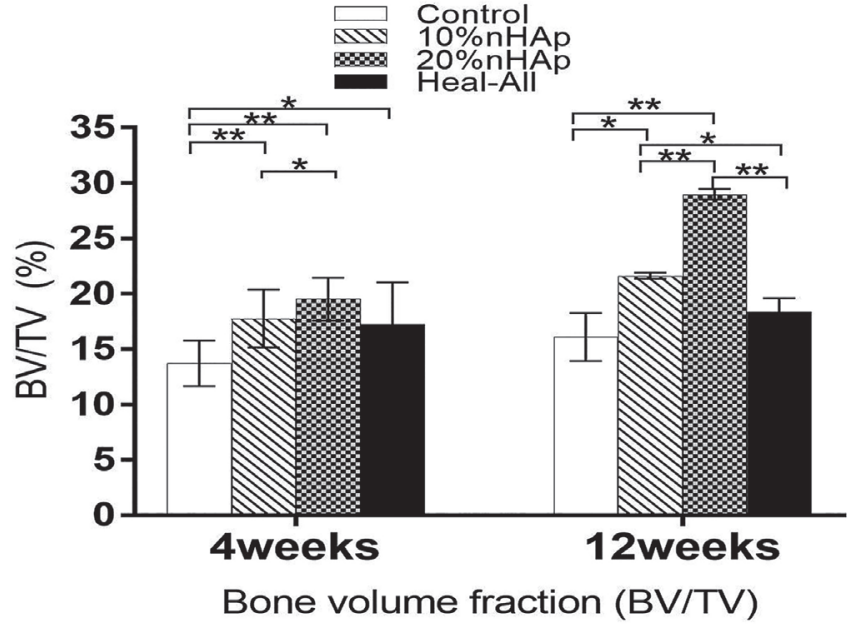

Figure 3. The percentage of new bone (BV/TV) between the four groups. Data are presented as means. Error bars represent mean \pm standard deviation, $n=3$, $* p<0.05, * * p<0.01, * * * p<0.001$.

values of the $10 \%$ and $20 \% \mathrm{nHAp} / \mathrm{PPC}$ and HEAL-ALL groups were higher than those of the control group at 4 and 12 weeks. And the BV/ TV values of $10 \%$ and $20 \% \mathrm{nHAp} / \mathrm{PPC}$ groups were higher than that of the HEAL-ALL group, while the $20 \% \mathrm{nHAp} / \mathrm{PPC}$ group had the highest value. Micro-CT analysis revealed that no significant difference was observed between the volume of mineralized tissues in nHAp/PPC group and HEAL-ALL group at 4 weeks (Fig. 3). But at the 12 weeks, both $10 \%$ and $20 \% \mathrm{nHAp} / \mathrm{PPC}$ groups significantly promoted mineralized tissue volume when compared to control and HEAL-ALL group (Fig. 3).

The Micro-CT bone morphometry datas at 4 and 12 weeks after surgery are shown in Table 2. The large values of Tb.Th and Tb.N indicate the maturity and stability of the bone structure. By contrast, if the value of $\mathrm{Tb} . \mathrm{Sp}$, which represents the degree of connectivity of the trabecular bone, is large, the arrangement of the bone structure is poor ${ }^{23)}$. It was found that the Tb.N and Tb.Th of the three groups were higher than those of the control group from Table 2 at postoperative weeks 4 and 12 . By contrast, the Tb.Sp of the three experimental groups was lower than 
J.Hard Tissue Biology Vol. 28(4): 371-376, 2019

Table 2. The micro-CT bone morphometry datas at 4 and 12 weeks after surgery

\begin{tabular}{rrcccc}
\hline & & Control & $10 \% \mathrm{nHAp} / \mathrm{PPC}$ & $20 \% \mathrm{nHAp} / \mathrm{PPC}$ & Heal-All \\
\hline \multirow{2}{*}{ Tb.N } & $4 \mathrm{w}$ & $0.834 \pm 0.072$ & $0.837 \pm 0.122^{\mathrm{b}}$ & $0.917 \pm 0.134^{\mathrm{c}}$ & $0.943 \pm 0.210$ \\
& $12 \mathrm{w}$ & $1.398 \pm 0.447$ & $1.546 \pm 0.554$ & $1.692 \pm 0.714$ & $1.414 \pm 0.396$ \\
\hline \multirow{2}{*}{ Tb.Th } & $4 \mathrm{w}$ & $0.097 \pm 0.004$ & $0.105 \pm 0.015$ & $0.116 \pm 0.010^{\mathrm{ab}}$ & $0.103 \pm 0.007^{\mathrm{a}}$ \\
& $12 \mathrm{w}$ & $0.163 \pm 0.012$ & $0.196 \pm 0.005^{\mathrm{ab}}$ & $0.200 \pm 0.009^{\mathrm{ab}}$ & $0.169 \pm 0.012^{\mathrm{a}}$ \\
\hline \multirow{2}{*}{ Tb.Sp } & $4 \mathrm{w}$ & $0.981 \pm 0.006$ & $0.856 \pm 0.010^{\mathrm{a}}$ & $0.800 \pm 0.071^{\mathrm{a}}$ & $0.766 \pm 0.106^{\mathrm{a}}$ \\
& $12 \mathrm{w}$ & $0.812 \pm 0.103$ & $0.595 \pm 0.086^{\mathrm{a}}$ & $0.652 \pm 0.144^{\mathrm{a}}$ & $0.659 \pm 0.080^{\mathrm{a}}$ \\
\hline
\end{tabular}

a: $\mathrm{p}<0.05$ vs. control; b: $\mathrm{p}<0.05$ vs. Heal-ALL; c: $\mathrm{p}<0.05$ vs. $10 \% \mathrm{nHAp} / \mathrm{PPC}$

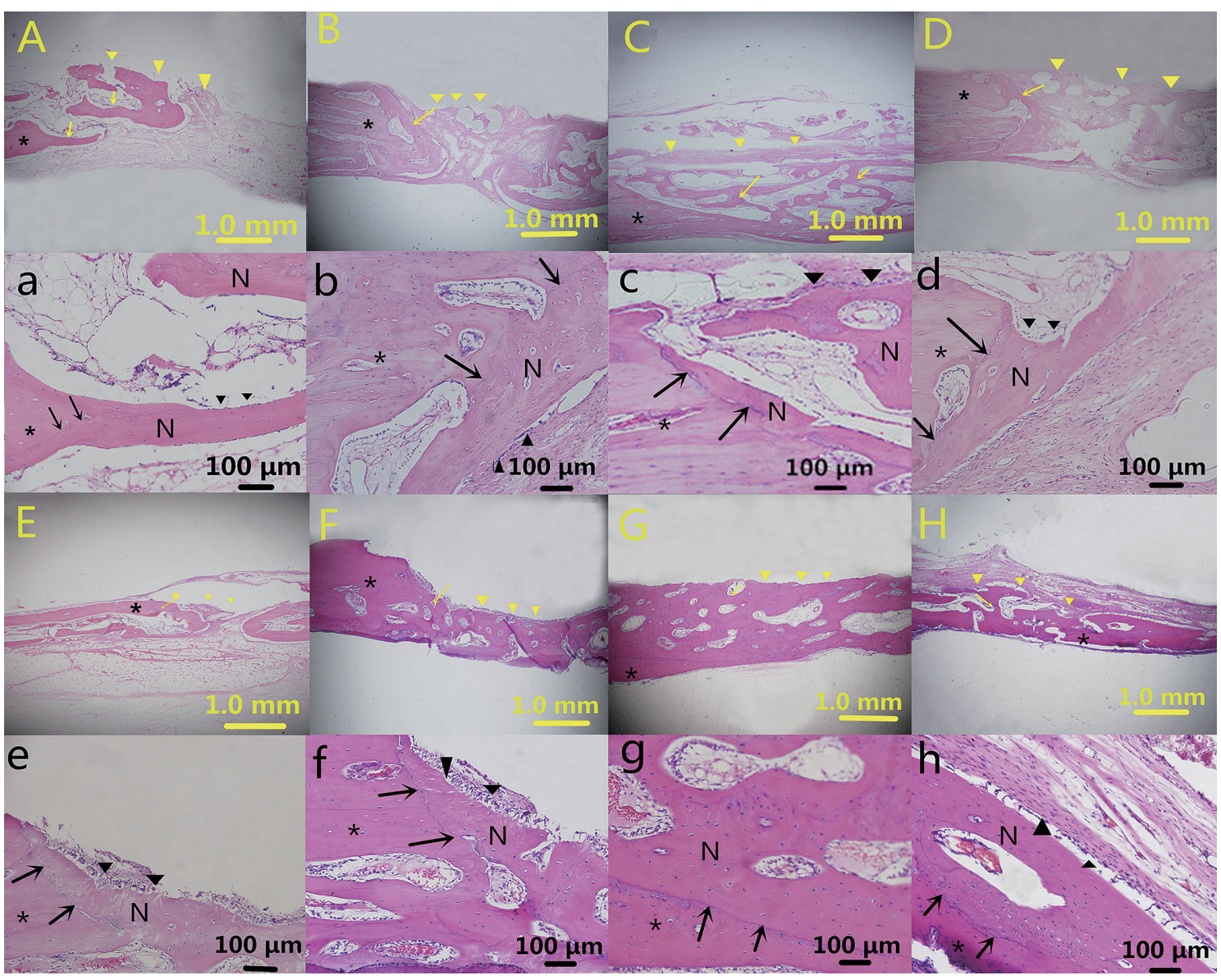

Figure 4. Histological specimens at 4 and 12 weeks of healing (Hematoxylin and Eosin staining). A, a: stainning images of control group at 4 weeks. B, b: stainning images of $10 \% \mathrm{nHAp} / \mathrm{PPC}$ group at 4 weeks. C, c: stainning images of $20 \% \mathrm{nHAp} / \mathrm{PPC}$ group at 4 weeks. D, d: stainning images of HEAL-ALL group at 4 weeks. E, e: stainning images of control group at 12 weeks. F, f: stainning images of $10 \% \mathrm{nHAp} / \mathrm{PPC}$ group at 12 weeks. G, g: stainning images of $20 \% \mathrm{nHAp} / \mathrm{PPC}$ group at 12 weeks. $\mathrm{H}$, h: stainning images of HEAL-ALL group at 12 weeks. Original magnification for A, B, C, D, E, F, G and H, ×4; and original magnification for a, b, c, d, e, f, g and h, $\times 20$. (*: original bone; N: new bone; yellow arrows: bone trabeculae; yellow arrowheads: bone defect; black arrows: reversal cement line; black arrowheads: osteoblastic rimming)

that of the control group. The $20 \% \mathrm{nHAp} / \mathrm{PPC}$ group showed better performance than the other groups, indicating that mature and well-connected bone tissue had formed.

\section{Histologic analysis}

The images of tissue sections stained by hematoxylin-eosin are displayed in Fig. 4. In all the tissue sections, necrotic tissue and inflammation or foreign body reaction was not observed. Four weeks after surgery, a large number of loose connective tissues were found in the control group, whereas a small amount of newly formed bone (denoted as yellow arrow) was seen at the edge of the defect area. More new bone appeared in the three experimental groups (denoted as yellow arrow). In the $10 \%, 20 \% \mathrm{nHAp} / \mathrm{PPC}$ and HEAL-ALL groups, bone-like structures (denoted as N) surrounded by osteoblasts (denoted as black arrowheads) were observed (Fig. 4). Meanwhile, 12 weeks after surgery, newly formed bone tissues was in the stage of remodeling and became more mature, and more lamellar bone formed (denoted as N). But for the control group in Fig. 4(E), we found that minimal new bone tissue could be observed. In the other three groups, the newly formed bone tissues had almost occupied the whole defect and more lamellar bone had formed and also became thicker. The $20 \% \mathrm{nHAp} / \mathrm{PPC}$ group had the most new bone at either 4 or 12 weeks. The results of hematoxylin-eosin staining 
was consistent with that of Micro-CT.

\section{Discussion}

The ideal GBR membrane should have good mechanical strength, biocompatibility, and biodegradability. In our previous experiments, we prepared a nHAp/PPC GBR membranes ${ }^{20)}$, where in one side of the biofilm is smooth and prevents soft tissue penetration and the other is porous and facilitates cell adhesion consistent with previous studies by other scholars ${ }^{24,25)}$. Moreover, we found in our previous study that the $10 \%$ and $20 \% \mathrm{nHAp} / \mathrm{PPC}$ groups had good mechanical properties and biocompatibility. Thus, the two groups were selected as the experimental groups in the present experiment, which aims to evaluate bone regeneration in rabbit calvarial defects covered with our proposed biofilm through Micro-CT and histological observation. HEAL-ALL membrane is a kind of GBR membrane, which is widely used in clinical practice in China. Its main component is collagen extracted from the dermal matrix of cattle skin. The HEAL-ALL membrane is supposed to degrade completely within 6 months according to the manufacture ${ }^{26}$. The success of the GBR procedure depends on space maintenance for bone formation at a sufficient period (of at least 6 weeks) ${ }^{27}$. Given that the stabilization of bone remodeling occurs approximately 3 months after surgery, bone regeneration in $\mathrm{nHAp} / \mathrm{PPC}$ was observed 4 and 12 week safter surgery.

In the present study, we performed three-dimensional reconstruction and data analysis on micro-CT data. The results showed that the volumes of the newly formed bone tissues of the $10 \%$ and $20 \% \mathrm{nHAp} / \mathrm{PPC}$ and the HEAL-ALL groups were larger than that of the control group at 4 weeks after surgery. And the BV/TV values of $10 \%$ and $20 \% \mathrm{nHAp} /$ PPC groups were higher than that of the HEAL-ALL group. Over time, although extent of bone formation increased in the four groups 12 weeks after surgery, the extent of bone formation in the $10 \%$ and $20 \% \mathrm{nHAp} /$ PPC groups was higher, and the $20 \% \mathrm{nHAp} / \mathrm{PPC}$ group had highest extent. The results of analysis of variance showed that the differences among the groups were most statistically significant. The reason that the new bone of the $10 \%$ and $20 \% \mathrm{nHAp} / \mathrm{PPC}$ groups was higher than that in the HEAL-ALL group is explained as follows: Although the HEALALL group has a bilayer structure composed of a compact and porous layer ${ }^{26)}$, it does not have $\mathrm{Ca}^{+}$ions and can only maintain the bone defect space during osteogenesis; this observation is consistent with a previous study $^{26)}$. The greater the value of Tb.T and Tb.N, the more mature and stable the bone structure. In contrast, Tb.Sp stands for the degree of connectivity of trabecular bone; if the value of Tb.Sp is bigger, the arrangement of the bone structure would be worse. The results of this experiment showed that the $10 \%$ and $20 \% \mathrm{nHAp} / \mathrm{PPC}$ and the HEAL-ALL groups had higher Tb.Th and Tb.N values and lower Tb.Sp values than the control group after 4 and 12 weeks of surgery. Furthermore, the $10 \%$ and $20 \% \mathrm{nHAp} / \mathrm{PPC}$ and the HEAL-ALL groups had higher volumes of regenerated bones covered by the membranes and the qualities of their newly formed bone tissues were better than those of the control group. This outcome may be attributed to the membranes, which resisted fibroblasts, maintained the space of the bone defects, and provided an anti-interference environment for osteogenesis.

Four and twelve weeks after surgery, no inflammation and necrotic tissues were detected in the rabbit parietal defect by histological observations. At 12 weeks, the presence of nHAp/PPC membrane was still visible to the naked eye. This phenomenon indicated that the prepared $10 \%$ and $20 \% \mathrm{nHAp} / \mathrm{PPC}$ films had good biocompatibility and sufficient degradation time to meet the requirements of bone regeneration. The hematoxylin-eosin stains showed that a large amount of loose connective tissue was present in the bone defect of the control group at 4 weeks and more new bone formation in the bone defects of the $10 \%$ and $20 \%$ nHAp/PPC and the HEAL-ALL groups, showing newly formed bone marrow cavities. At 12 weeks, a small amount of new bone tissue formed in the control group, whereas the newly formed bone tissues in the $10 \%$ and $20 \% \mathrm{nHAp} / \mathrm{PPC}$ and the HEAL-ALL groups were numerous and thicker. The extent of bone formation in the $\mathrm{nHAp} / \mathrm{PPC}$ group was obvious possibly because of $\mathrm{Ca}^{+}$ions production during the degradation of the nHAp/PPC membrane. The process provided the raw material for bone formation. Furthermore, the $20 \% \mathrm{nHAp} / \mathrm{PPC}$ group contained many $\mathrm{Ca}^{+}$ions, and thus generated a large volume of bone tissues. The results of hematoxylin-eosin staining were consistent with the results of micro-CT.

Similar to the HEAL-ALL membrane, the synthesized nHAp/PPC membrane had good biocompatibility and good effect on GBR. The micro-CT and histology analysis results showed that the $20 \% \mathrm{nHAp} / \mathrm{PPC}$ group had good effect on bone regeneration, and these results are consistent with our in vitro results ${ }^{21)}$. However, to perform further studies to explore the mechanism of bone regeneration before the clinical applications of nHAp/PPC membrane is still necessary.

\section{Acknowledgments}

The authors would like to acknowledge the Changchun Institute of Applied Chemistry Chinese Academy of Sciences for providing PPC samples freely. This work was supported by the Fund of Science and Technology Department of Jilin Province, China (No.20170204009YY), the Fund of Science \& Technology Department of Changchun City, China (No.17YJ001), the Project of the Ministry of Science and Technology of China (No.2016YFE0128500), the Fund of Development and Reform Commission of Jilin Province, China (No.2016C048-3).

\section{Conflict of Interest}

The authors have declared that no COI exists.

\section{References}

1. Abdulai A, Iddrissu M and Dakurah T. Cranioplasty using polymathyl methacrylate implant constructed from an alginate impression and wax elimination technique. Ghana Med J 40: 18-21, 2006

2. Böker DK, Schultheiss R and Probst EM. Radiologic long-term results after cervical vertebral interbody fusion with polymethyl methacrylat (PMMA). Neurosurg Rev 12: 217-221, 1989

3. Rakhmatia YD, Ayukawa Y, Furuhashi A and Koyano K. Current barrier membranes: titanium mesh and other membranes for guided bone regeneration in dental applications. J Prosthodont Res 57: 3-14, 2013

4. Benic GI and HammerleCH. Horizontal bone augmentation by means of guided bone regeneration. Periodontol 66: 13-40, 2014

5. Cowan CM, Shi Y-Y, Aalami OO, Chou Y-F, Mari C, Thomas R, Quarto N, Contag CH, Wu B and Longaker MT. Adipose-derived adult stromal cells heal critical-size mouse calvarial defects. Nat Biotechnol 22: 560-567, 2004

6. Dahlin C, Sennerby L, Lekholm U, Linde A and Nyman S. Generation of new bone around titanium implants using a membrane technique: an experimental study in rabbits. Int J Oral Maxillofac Implants 4: 19-25, 1989

7. Retzepi M and Donos N. Guided bone regeneration: biological principle and therapeutic applications. Clin Oral Implants Res 21: 567576,2010

8. Liu J and Kerns DG. Mechanisms of guided bone regeneration: a review. Open Dent J 8: 56-65, 2014 
9. Langer B, Langer L and Sullivan RM. Vertical ridge augmentation procedure using guided bone regeneration, demineralized freezedried bone allograft, and miniscrews: 4- to 13-year observations on loaded implants. Int J Periodontics Restorative Dent 30: 227-235, 2010

10. Chiapasco M and Zaniboni M. Clinical outcomes of GBR procedures to correct peri-implant dehiscences and fenestrations: a systematic review. Clin Oral Implants Res 20: 113-123, 2009

11. Zitzmann NU, Naef R and Scharer P. Resorbable versus nonresorbable membranes in combination with Bio-Oss for guided bone regeneration. Int J Oral Maxillofac Implants 12: 844-852, 1997

12. Kim G, Ree M, Kim H, Kim IJ, Kim JR and Lee JI. Biological affinity and biodegradability of poly(propylene carbonate) prepared from copolymerization of carbon dioxide with propylene oxide. Macromol Res 16: 473-480, 2008

13. Klaus S, Lehenmeier MW, Anderson CE, StephanKlaus and Rieger B. Recent advances in $\mathrm{CO}_{2}$ /epoxide copolymerization-New strategies and cooperative mechanisms. Coordin Chem Rev 255: 14601479, 2011

14. Chen LJ, Qin YS, Wang XH, Zhao XJ and Wang FS. Plasticizing while toughening and reinforcing poly(propylene carbonate) using low molecular weight urethane: Role of hydrogenbonding interaction. Polymer 52: 4873-4880, 2011

15. Dong HP, Kan TG, Yun KL and Kim WN. Effect of multi-walled carbon nanotube dispersion on the electrical and rheological properties of poly(propylene carbonate)/poly(lactic acid)/multi-walled carbon nanotube composites. J Mater Sci 48: 481-488, 2013

16. Fox K1, Tran PA and Tran N. Recent advances in research applications of nanophase hydroxyapatite. Chemphyschem 13: 2495-2506, 2012

17. Hench LL and Polak JM. Third-generation biomedical materials. Science 295: 1014-1017, 2002
18. Habibovic P, Kruyt MC, Juhl MV, Clyens S, Martinetti R, Dolcini L Theilgaard $\mathrm{N}$ and van Blitterswijk CA. Comparative in vivo study of six hydroxyapatite-based bone graft substitutes. J Orthop Res 26: 1363-1370, 2008

19. Kokubo T and Takadama H. How useful is SBF in predicting in vivo bone bioactivity?. Biomaterials 27: 2907-2915, 2006

20. Lan CJ, Xiang XC, Chu SL, Wei W, He X and Li J. Preparation, characterization and properties of $\mathrm{nHAp} / \mathrm{PPC}$ membrane. J Hard Tissue Biol 27: 295-302, 2018

21. Lan CJ, Xiang XC, Gao X, Sun D, Pan YS and Li J. Cellular Compatibility Analysis of nHAp/PPC Membrane. J Hard Tissue Biol 28: 31-36, 2019

22. Christiansen BA. Effect of micro-computed tomography voxel size and segmentation method on trabecular bone microstructure measures in mice. Bone Rep 5: 136-140, 2016

23. Wu WY, Li BW, Liu YH, Wang XZ and Tang L. Effect of Multilaminate small intestinal submucosa as a barrier membrane on bone formation in a rabbit mandible defect model. Biomed Res Int 2018: $1-11,2018$

24. Hwang JW, Kim S, Kim SW and Lee JH. Effect of extracellular matrix membrane on bone formation in a rabbit tibial defect model. BioMed Res Int 2016: 1-8, 2016

25. Tu Y, Chen C, Li YB, Hou Y, Huang M and Zhang L. Fabrication of nano-hydroxyapatite/chitosan membrane with asymmetric structure and its applications in guided bone regeneration. Bio-Med Mater Eng 28: 223-233, 2017

26 Guo Y, Liu WW, Ma SS, Wang J, Zou JT, Liu ZZ, Zhao JH and Zhou YM. A preliminary study for novel use of two Mg alloys (WE43 and Mg3Gd). J Mater Sci Mater Med 27: 82-95, 2016

27. Olaechea A, Mendoza-Azpur G, Valdivia E and Perini GR. Biodegradation of three different collagen membranes: A histological study. J Osseointegra 8: 15-19, 2016 